\title{
Unoperated ovarian endometriomas and responsiveness to hyperstimulation
}

\author{
Laura Benaglia ', Roberta Pasin 1,2, Edgardo Somigliana I,*, \\ Paolo Vercellini I,2, Guido Ragni ', and Luigi Fedele I,2
}

\author{
'Department of Obstetrics, Gynecology and Neonatology, Fondazione Cà Granda, Ospedale Maggiore Policlinico, Via M. Fanti, 6, 20122 \\ Milan, Italy ${ }^{2}$ Università degli Studi di Milano, Milan, Italy \\ *Correspondence address. Tel: +39-2-57994303; Fax: +39-2-57994302; E-mail: dadosomigliana@yahoo.it \\ Submitted on December 8, 2010; resubmitted on February 6, 2011; accepted on March 7, 2011
}

\begin{abstract}
BACKGROUND: There is a growing consensus that ovarian reserve is reduced after surgical excision of ovarian endometriomas. However, it remains to be fully clarified whether this damage precedes or follows surgery. In order to further elucidate this aspect, we evaluated ovarian responsiveness to hyperstimulation in women selected for IVF with unilateral unoperated endometriomas. The main aim of this study was to compare the number of developing follicles in the affected ovary with that in the contralateral intact gonad as a control.
\end{abstract}

METHODS: Patients selected for IVF who were diagnosed with one or more monolateral endometriomas (diameter $<4 \mathrm{~cm}$ ) and who did not undergo previous ovarian surgery were retrospectively identified. We compared the number of follicles (diameter $\geq 1 \mathrm{Imm}$ ) and the number of co-dominant follicles (diameter $>15 \mathrm{~mm}$ ) on the day of hCG administration in the affected and intact ovaries.

RESULTS: Among the 84 women selected, the median interquartile range (IQR) number of follicles $\geq 11 \mathrm{~mm}$ in the affected and intact ovaries was $5(3-7)$ and $5(3-8)$, respectively $(P=0.36)$. The median (IQR) number of co-dominant follicles in the affected and intact ovaries was $3(2-4)$ and $3(2-5)$, respectively $(P=0.48)$. The number of co-dominant follicles was lower in the affected ovary in 36 cases (43\%, 95\% confidence interval: $32-53 \%)$. We also failed to identify any statistically significant difference between the two ovaries when evaluating data according to the number of cysts, their dimension, the dose of gonadotrophins used or the number of oocytes retrieved.

CONCLUSIONS: In women selected for IVF, the presence of an endometrioma does not markedly affect responsiveness to hyperstimulation.

Key words: endometriosis / endometrioma / IVF / ovarian reserve

\section{Introduction}

There is growing and consistent evidence demonstrating that ovarian reserve is affected following surgical excision of ovarian endometriomas (Garcia-Velasco and Somigliana, 2009). The rate of spontaneous ovulation is lower in operated ovaries (Loh et al., 1999; Candiani et al., 2005), serum levels of anti-mullerian hormone (AMH) decrease after surgery (Chang et al., 20l0) and responsiveness to hyperstimulation is reduced (Gupta et al., 2006). In women selected for IVF who have undergone excision of unilateral endometriomas, the number of developing follicles and the number of oocytes retrieved in the operated gonad are halved compared with the contralateral intact ovary (Loh et al., 1999; Ho et al., 2002; Somigliana et al., 2003; Ragni et al., 2005; Alborzi et al., 2006; Duru et al., 2007; Almog et al., 20l0). The few available data regarding women operated on for bilateral endometriomas are in line with this evidence. In these patients, IVF outcome is significantly impaired (Esinler et al., 2006; Somigliana et al., 2008) and, albeit rare, some cases of premature menopause following surgery have been reported (Busacca et al., 2006; Di Prospero and Micucci, 2009).

The debate on this point is now moving to the causes surrounding this damage. Of relevance here is that there is scant but important data supporting the view that the damage may in part precede the operation. Using pathological sections of the ovarian cortex surrounding ovarian benign neoplasms, Maneschi et al. (1993) found a reduced follicular number and activity antecedent to surgery in endometriomas when compared with teratomas or benign cystadenomas, suggesting that the disease per se may be detrimental to the ovary. Data on spontaneous ovulation in unoperated women with monolateral endometriomas tend to confirm these findings. Two recent independent papers showed that the rate of ovulation in the affected ovaries is significantly lower than in the contralateral ovaries (Horikawa et al., 2008; Benaglia et al., 2009). On the other hand, Almog et al. (201 I) recently reviewed 81 unoperated women, with monolateral endometriomas,

(C) The Author 2011. Published by Oxford University Press on behalf of the European Society of Human Reproduction and Embryology. All rights reserved. 
who underwent IVF and they failed to document a reduced responsiveness in the affected ovary. This result is of utmost interest considering that ovarian responsiveness to hyperstimulation is currently considered the best surrogate tool to evaluate ovarian reserve. Of note, however, the data from (Almog et al., 20l I) are in contrast with a similar paper published by our group in 2006 showing a $25 \%$ [95\% confidence interval $(\mathrm{Cl}): 6-44 \%$ ] reduction in the number of developing follicles in the affected gonad (Somigliana et al., 2006).

For the present study, we deemed it important to further explore this topic. With this aim, we present an extension of our initial data (Somigliana et al., 2006) by reporting on a series of 84 unoperated women with unilateral endometriomas selected for IVF. The main aim of the study was to compare the number of developing follicles in the affected ovary using the contralateral intact gonad as a control.

\section{Materials and Methods}

Data from IVF-ICSI cycles performed at the Infertility Unit of the Department of Obstetrics and Gynecology of the Fondazione Cà Granda, Ospedale Maggiore Policlinico between January 2000 and July 2009 were retrospectively reviewed. The study focused on women with unoperated monolateral endometriomas, and it aimed at comparing ovarian responsiveness in the affected and contralateral intact ovaries. The outcome chosen was the number of developing follicles. With this aim, we included patients who were diagnosed with one or more monolateral endometrio$\mathrm{ma}(\mathrm{s})$ and who did not undergo previous ovarian surgery. Specifically, inclusion criteria were as follows: (i) women selected for IVF-ICSI cycles who underwent ovarian hyperstimulation, (ii) age $\leq 40$ years, (iii) ecographic diagnosis of one or more unilateral endometrioma(s) with a diameter $<4 \mathrm{~cm}$, (iv) presence of the endometrioma(s) confirmed at least twice at a 2 months interval and (v) development of at least three follicles with a mean diameter $\geq 11 \mathrm{~mm}$. Criterion Number 3 was decided based on ESHRE guidelines suggesting surgical removal of endometriomas with a diameter $\geq 4 \mathrm{~cm}$ in women selected for IVF (Kennedy et al., 2005). Criterion Number 4 was added to rule out haemorrhagic functional cysts. Criterion Number 5 was decided to exclude women whose ovarian reserve was compromised for reasons unrelated to the presence of ovarian endometriomas. Exclusion criteria were as follows: (i) previous ovarian surgery for endometriomas and/or non-endometriotic benign ovarian cysts, (ii) diagnosis of bilateral endometriomas or (iii) cancelled cycles due to hyper or low response. Patients were included only for their first treatment cycle reaching oocyte retrieval. The study was approved by the local Institutional Review Board. All women in our Unit routinely give a general consent for the use of their data for research purposes. No specific informed consent for the present analysis was obtained since this is a retrospective study.

Ecographic diagnosis of endometriomas was performed as previously reported (Mais et al., 1993; Kurjak and Kupesic, 1994). Many studies have validated the non-surgical diagnosis of these cysts with transvaginal ultrasound (Savelli, 2009). The precise protocol of treatment and monitoring of IVF-ICSI cycles in our Unit is reported elsewhere in detail (Somigliana et al., 2003; Ragni et al., 2005). In this context, it is of interest that an ultrasound scan was systematically performed on the day of hCG administration (when two or more leading follicles had mean diameter $>18 \mathrm{~mm}$ ). On this occasion, the number and dimension of all follicles with a mean diameter $\geq 11 \mathrm{~mm}$ were recorded. These variables were scheduled separately for the two ovaries. The diameter of endometriomas and follicles was calculated as the mean of three perpendicular diameters.

Data analysis was performed using the Statistics Package for Social Sciences (SPSS 15.0, Chicago, IL, USA). The primary outcome was the number of follicles with a mean diameter $\geq 11 \mathrm{~mm}$. Since an assumption of normal distribution could not be reliably done, the analyses were performed using non-parametric statistics. Paired Wilcoxon test was used to investigate differences between affected and contralateral intact ovaries. Data were reported as median [interquartile range (IQR)]. The frequency of silent ovaries (absence of follicular growth) was compared between the two groups using the MacNemar test. A $P$-value $<0.05$ was considered as statistically significant. A binomial distribution model was used to determine the $95 \% \mathrm{Cl}$ of proportions. The null hypothesis stated that the number of follicles did not differ. Based on data emerging from our initial study (Somigliana et al., 2006) and using the conventional types I and II error of 0.05 and 0.20 , a sample size of at least 80 women would allow us to detect differences corresponding to a condition of reduced responsiveness in the affected gonad in $>60 \%$ of cases (considering as a null hypothesis a probability of reduced responsiveness of $50 \%$ ).

\section{Results}

There were 84 women fulfilling our inclusion and exclusion criteria. The mean \pm SD age, BMI, duration of infertility and Day 3 serum FSH were $35.0 \pm 3.8$ years, $21.5 \pm 3.0 \mathrm{~kg} / \mathrm{m}^{2}, 3.7 \pm 2.4$ years and $7.8 \pm 2.6 \mathrm{IU} / \mathrm{ml}$, respectively. Of the 84 women, 12 (I4\%) had a previous pregnancy. The indication to IVF was endometriosis in 36 (43\%) women, whereas in the remaining 48 (57\%) there was also a concomitant male factor. A single endometrioma was documented in 74 (88\%) women. The remaining 10 (12\%) had two or more cysts. Endometriomas were located in the right and left side in 30 (36\%) and 54 (64\%) cases, respectively. The mean \pm SD diameter of these lesions was $21 \pm 8 \mathrm{~mm}$. Following the documented IVF cycle, 30 women underwent laparoscopic removal of the cysts in our Unit. The diagnosis of endometriosis was confirmed in all of them. IVF data was as follows: 59 (70\%) women were treated with a long protocol of stimulation (daily $\mathrm{GnRH}$ agonists initiated in the mid-luteal phase, 2-3 weeks before starting the gonadotrophins), whereas 25 (30\%) received gonadotrophins from the third day of the cycle and then added daily $\mathrm{GnRH}$ antagonists to prevent spontaneous ovulation after the detection of the leading follicle with a mean diameter of 13-14 mm. None received pre-IVF treatment with oral contraceptives or progestins. The mean \pm SD total dose of recombinant FSH administered, the duration of stimulation and the number of oocytes retrieved were $2714 \pm 1292 \mathrm{IU}, \quad 11.0 \pm 2.1$ days and $7.2 \pm 4.8$, respectively. The median concentration of spermatozoa in couples retrieving oocytes was $62 \times 10^{6} / \mathrm{ml}\left(\right.$ IQR: $\left.29-101 \times 10^{6} / \mathrm{ml}\right)$. The median fertilization rate was $100 \%$ (IQR: $52-100 \%$ ). No embryos were available for transfer in five (6\%) cases. In women undergoing embryo transfer, the mean \pm SD number of embryos transferred was $2.2 \pm 0.8$. There were 24 (29\%) women who became pregnant. The implantation rate was $23 \%$.

The median (IQR) of the total number of follicles (diameter $\geq 11 \mathrm{~mm}$ ) in the affected and intact ovaries was $5(3-7)$ and 5 (38), respectively $(P=0.36)$. The total number of follicles was lower in the affected ovary in 42 cases $(50 \%, 95 \% \mathrm{Cl}$ : $39-61 \%)$. When specifically considering the co-dominant follicles (diameter $>15 \mathrm{~mm}$ ), the median (IQR) number in the affected and intact ovaries was $3(2-4)$ and $3(2-5)$, respectively $(P=0.48)$. The number of co-dominant follicles was lower in the affected ovary in 36 cases (43\%, 95\% Cl: $32-53 \%)$. These results are illustrated in Figure I. There was an absence of follicular growth (silent ovaries) 
in two affected ovaries (2.4\%) and three contralateral intact gonads (3.6\%) $(P=1.00)$.

Data were also analysed according to the characteristics of the endometrioma(s) and the responsiveness to ovarian hyperstimulation (Table I). We failed to document any statistically significant impact when evaluating separately the affected and intact ovaries for the number of cysts, their dimension, the dose of gonadotrophins used or the number of oocytes retrieved. The analysis was also repeated considering separately women who received $\mathrm{GnRH}$ agonists and those who received $\mathrm{GnRH}$ antagonists but no substantial difference emerged (data not shown).

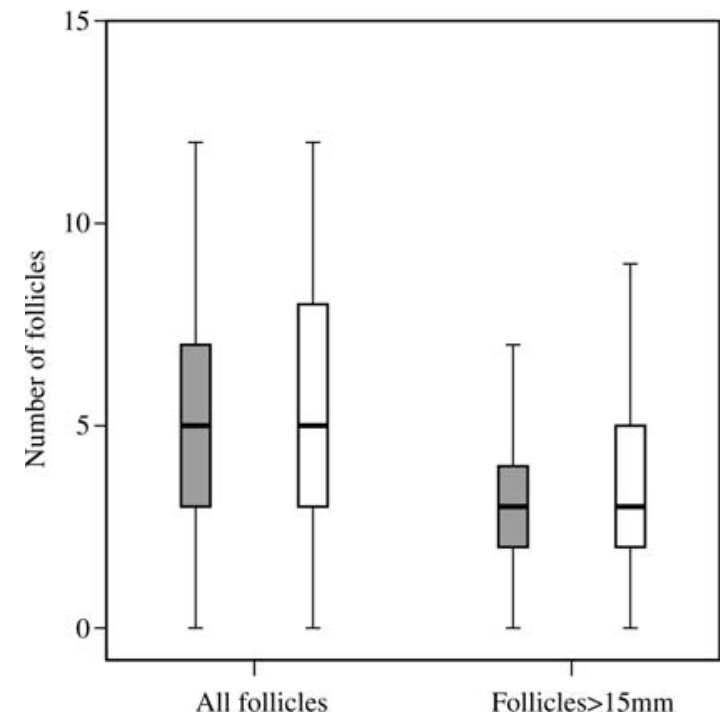

Figure I Number of follicles in ovaries with (grey boxes) and without (white boxes) endometriomas. The total number of follicles and the number of co-dominant follicles was similar in the two groups. The corresponding $P$-values were $P=0.36$ and $P=0.48$, respectively.

\section{Discussion}

In the present study, we failed to document a statistically significant difference in ovarian responsiveness to hyperstimulation in ovaries with endometriomas. The total number of follicles and the number of co-dominant follicles were similar in affected and intact gonads. This result is inline with the recent paper of Almog et al. (20I I) but in contrast with our initial data (Somigliana et al., 2006). There are at least two explanations for this incongruence. Firstly, the relatively small sample size of our initial study (56 IVF cycles performed in 36 women) exposed our results to a type I error. Secondly, in our initial study, the statistically significant difference between affected and intact ovaries emerged only when applying an analysis per cycle. Indeed, when focussing exclusively on the first IVF cycle (analysis per patient), there was a trend towards a lower response, but the difference did not reach statistical significance $(P=0.09)$. This is an important point since, in fact, we cannot exclude that the inclusion of more than one cycle per patient may have led us to overestimate the detrimental effects of endometriomas by selecting women with a worse prognosis. It is noteworthy that in the present study, we included only the first treatment cycle per patient.

A major strength of our present study is the relatively large sample size which, in fact, allows us to limit the possibility of a relevant type II error. Of additional interest here is that subgroup analyses focusing on the dimension, the number of the cysts and the ovarian responsiveness failed to identify subgroups of cases with significant damage. The lack of any trend for a gradient effect with the dimension and number of the endometriomas is particularly enlightening. The demonstration of a biological gradient over and above that of the dichotomous response is considered under the criterion of strength for establishing causality (Vigano et al., 2007). Overall, despite being inevitably exposed to a more important type II error, our subgroups analyses further supports our general conclusions.

Considering possible limitations, it has first to be recognized that a histological confirmation of the diagnosis of endometriosis is missing in the majority of cases. Only a subset of the patients successively underwent surgical excision of the cyst. On the other hand, due to their characteristic echogenic appearance, endometriomas can usually be easily distinguished from other ovarian cysts. Sensitivity and specificity

Table I Number of follicles according to the characteristics of the endometrioma(s) and the responsiveness to ovarian hyperstimulation.

\begin{tabular}{|c|c|c|c|c|c|c|c|c|}
\hline \multirow[t]{2}{*}{ Variables } & & \multirow[t]{2}{*}{ No. of cycles } & \multicolumn{3}{|c|}{ Number of follicles $\geq \mathrm{II} \mathrm{mm}$} & \multicolumn{3}{|c|}{ Number of follicles $>15 \mathrm{~mm}$} \\
\hline & & & Affected ovary & Intact ovary & $P$ & Affected ovary & Intact ovary & $P$ \\
\hline Number of cysts & $\begin{array}{r}1 \\
\geq 2\end{array}$ & $\begin{array}{l}74 \\
10\end{array}$ & $\begin{array}{l}4(3-7) \\
6(5-8)\end{array}$ & $\begin{array}{r}5(3-8) \\
6(2-10)\end{array}$ & $\begin{array}{l}0.22 \\
0.54\end{array}$ & $\begin{array}{l}3(2-4) \\
4(2-4)\end{array}$ & $\begin{array}{l}3(2-5) \\
4(2-6)\end{array}$ & $\begin{array}{l}0.49 \\
0.81\end{array}$ \\
\hline Diameter of the cysts ${ }^{\mathrm{a}}$ & $\begin{array}{l}<20 \mathrm{~mm} \\
\geq 20 \mathrm{~mm}\end{array}$ & $\begin{array}{l}34 \\
40\end{array}$ & $\begin{array}{l}5(3-7) \\
4(2-7)\end{array}$ & $\begin{array}{l}4(4-8) \\
5(3-7)\end{array}$ & $\begin{array}{l}0.46 \\
0.32\end{array}$ & $\begin{array}{l}3(2-4) \\
3(2-5)\end{array}$ & $\begin{array}{l}3(2-5) \\
3(2-5)\end{array}$ & $\begin{array}{l}0.41 \\
0.84\end{array}$ \\
\hline Total IU of rFSH used & $\begin{array}{l}<2500 \\
\geq 2500\end{array}$ & $\begin{array}{l}39 \\
45\end{array}$ & $\begin{array}{l}5(4-7) \\
4(2-7)\end{array}$ & $\begin{array}{l}6(4-9) \\
4(2-6)\end{array}$ & $\begin{array}{l}0.13 \\
0.94\end{array}$ & $\begin{array}{l}3(2-4) \\
3(2-4)\end{array}$ & $\begin{array}{l}4(2-6) \\
3(2-5)\end{array}$ & $\begin{array}{l}0.16 \\
0.75\end{array}$ \\
\hline Number oocytes retrieved & $\begin{array}{l}\leq 5 \\
>5\end{array}$ & $\begin{array}{l}40 \\
44\end{array}$ & $\begin{array}{l}4(2-6) \\
6(4-8)\end{array}$ & $\begin{array}{l}4(2-6) \\
6(4-9)\end{array}$ & $\begin{array}{l}0.87 \\
0.18\end{array}$ & $\begin{array}{l}3(2-4) \\
4(3-5)\end{array}$ & $\begin{array}{l}3(2-4) \\
4(3-6)\end{array}$ & $\begin{array}{l}0.92 \\
0.28\end{array}$ \\
\hline
\end{tabular}

Data are reported as median (IQR).

${ }^{a}$ Women with more than one cyst were excluded. 
of transvaginal ultrasound have been reported to be $84-100 \%$ and 90-100\%, respectively (Mais et al., 1993; Kurjak and Kupesic, 1994; Eskenazi et al., 200I; Garcia-Velasco et al., 2004). Moreover, the decision to include only cysts which were documented on at least two occasions and at least two menstrual cycles apart should have eliminated the possibility of enrolling women with haemorrhagic cysts. Finally, it is noteworthy that a diagnosis of endometriosis was confirmed in all operated cases, supporting the reliability of the sonographic diagnosis.

A second possible limitation of the study is that we selectively recruited women with an indication for IVF: these women may not properly reflect the whole population of women with ovarian endometriomas. The dimension of the endometriomas in our study was small, with a mean diameter of $21 \mathrm{~mm}$, and pain symptoms were generally modest or null (severe pain symptoms in patients with an endometrioma was an indication to surgery). The main advantage of recruiting women selected for IVF was to obtain data from ovarian responsiveness to hyperstimulation since this outcome is currently considered the best surrogate tool to measure ovarian reserve (Broekmans et al., 2006). One may however argue that, even if less accurate, the use of alternative markers of ovarian reserve in an unselected population would have been more informative. In fact, serum levels of $\mathrm{FSH}$, inhibin $\mathrm{B}$ or $\mathrm{AMH}$ and the number of ovarian antral follicles count (AFC) are commonly recognized as reliable tools to assess ovarian reserve. However, these markers have some limits. The serum dosages do not provide independent information for the two gonads. In women with monolateral disease, the intact gonad may properly supply hormones despite the altered function of the affected gonad (Garcia-Velasco and Somigliana, 2009). In order to provide conclusive hormonal information, one should thus aim at exclusively recruiting women with bilateral endometriomas. Again, however, these women would represent a highly selected group. AFC appears to be a more attractive option since it allows the collection of data independently for the two ovaries. As in the present study, it offers the precious possibility of focusing on women with monolateral disease, thus allowing us to control for inter-patient variability. In our view, interesting insights may be provided by future studies using this study design in unselected women. One possible concern in this context is, however, the accuracy of AFC in the presence of an ovarian endometrioma. To date, no studies have indeed been published on this point. Given that ovarian responsiveness remains the best surrogate tool to measure ovarian reserve (Broekmans et al., 2006), one may argue that the use of other markers of ovarian responsiveness such as the number of oocytes retrieved or the number developing embryos may be more suitable than follicle count. Unfortunately, these two variables were not recorded separately per ovary in our unit. They are thus not available. In this regard, it has to be pointed out, however, that the number of oocytes retrieved and the number of developing embryos may also not properly reflect ovarian reserve since they may be influenced by other independent variables. Of particular relevance here is that the presence of the endometriomas may be a mechanical obstacle to the pick-up and, consequently, the number of oocytes retrieved or developing embryos may be lower in affected gonads, but this would not be consequent to an altered ovarian responsiveness.

A further limit is the assumption that, in unaffected women, ovarian responsiveness is similar in the two ovaries. This may not be entirely true. A recent paper suggests that the response to hyperstimulation is mildly superior in the right ovary (Lan et al., 2010). However, we do not estimate that this may have influenced our results considering that endometriomas are mostly located in the left ovary. This would be expected to overestimate the impact of the endometrioma. In contrast, here, we failed to detect any difference.

Finally, one may hypothesize that ovarian endometriomas are not the unique form of endometriosis that can impair ovarian responsiveness. Other forms of the disease such as adhesions or ovarian superficial implants may also be detrimental. In fact, the presence of these lesions cannot be reliably documented in the context of the present study since only sonographic findings were used. All women would have to undergo a diagnostic laparoscopy before the IVF cycle to have reliable information on adhesions or ovarian superficial implants and this is not ethically acceptable. Given, however, the lack of differences between the two ovaries, this possible limitation loses relevance. It is noteworthy that adhesions and superficial implants are expected to be more frequent in the ovary with the endometrioma. It cannot be reasonably argued that the lack of difference between the two gonads would be due to a bilateral impairment with the endometrioma acting on one ovary and adhesions and superficial lesions acting on the contralateral gonad.

Our data are partly in contrast with the scant available evidence. Using pathological sections of the ovarian cortex surrounding ovarian benign neoplasms, Maneschi et al. (1993) showed that, in the case of endometriotic cysts, the damage may in part precede surgery. These authors documented morphologic patterns similar to those of the normal ovarian cortex in the cortical tissue surrounding mature teratomas, benign cystomas and endometriomas in 92, 77 and 19\% $(P<0.01)$ of specimens, respectively. In a recent study from our group focusing on an unselected population of women with unilateral endometriomas, we observed that spontaneous ovulation occurs less frequently in the affected gonad. Specifically, this event was documented in about one-third of cases (22 out of 70 cases corresponding to $31 \%$; 95\% Cl: 22-43\%) (Benaglia et al., 2009). Horikawa et al. (2008) reported a similar result. On the other hand, our present results are in line with those reported by Almog et al. (20lI). These authors reported that the number of antral follicles and oocytes retrieved in the endometrioma-containing ovary $(6.0 \pm 0.4$ and $7.7 \pm 1.0$, respectively) and in the opposite ovary (6.1 \pm 0.5 and $8.5 \pm 0.9$, respectively) were similar. Despite an apparent incongruence, these findings may not be in contrast. The observation that follicles surrounding the capsule of the endometrioma are reduced may correspond to a mild insult to the overall reserve of primordial follicles of that ovary. Similarly, it cannot be excluded that a mild reduction in ovarian reserve may determine a marked reduction in the rate of ovulation. Contrary to the previous evidence, our study design allows us to define a more precise estimate of the magnitude of the effect of the presence of an endometrioma and overall supports the view that the effect is, if any, mild. However, further studies are warranted in unselected women with endometriomas to draw definite conclusions. In fact, our considerations are limited to a selected population of patients undergoing IVF and may be not generalized to all women with unilateral endometriomas. Finally, a further concern is the temporality of exposure and outcome. One may argue that the injury to ovarian reserve may take place progressively over time. Albeit theoretical, this may represent a 
source of confounding. We cannot rule out the possibility that the time period between development of the endometrioma and assessment may differ in women selected for IVF and in those operated for endometriomas.

Even though our study supports the idea that the presence of an endometrioma is unremarkable in terms of ovarian responsiveness, inferences regarding oocyte quality cannot be drawn. This is a critical point as follicular development and oocyte quality may be impaired by the proximity of an endometrioma. Although the pregnancy rate in our case series was similar to the rate of success observed for other indications during the same study period, our data do not definitely clarify this aspect. The only available randomized controlled trial on this topic tends to support the notion that ovarian endometriomas have no effect on oocyte quality. Indeed, the fertilization rate and the pregnancy rate were not affected in the unoperated cases when compared with those who underwent surgery prior to IVF (Demirol et al., 2006). Further evidence is, however, required to definitely clarify the impact of ovarian endometriomas on oocyte quality. In particular, prospective studies comparing morphological characteristics and the rate of fertilization of oocytes retrieved from affected and contralateral intact ovaries would be of interest in this regard. Alternatively, and as previously mentioned, efforts should aim at recruiting women with bilateral unoperated endometriomas. Evaluation of serum levels of $A M H$ in this group may be informative since this has been shown to reflect oocyte quality (Nelson et al., 2009) while removing the component of the health of the ovary.

In conclusion, the presence of small endometriomas $(<4 \mathrm{~cm})$ at the time of IVF does not markedly affect responsiveness to hyperstimulation, thus suggesting a mild, if any, impact on ovarian reserve. The impact of larger or symptomatic cysts remains to be clarified along with the possible effects on oocyte quality.

\section{Authors' roles}

L.B., E.S. and G.R. conceived and designed the study. R.P. acquired the data and participate to the analysis. P.V. and L.F. gave substantial contribution to the analysis and interpretation of the data. L.B. wrote the first draft and all the other authors revised it critically adding important intellectual content. All authors gave final approval to the final version of the manuscript.

\section{References}

Alborzi S, Zarei A, Alborzi S, Alborzi M. Management of ovarian endometrioma. Clin Obstet Gynecol 2006;49:480-49I.

Almog B, Sheizaf B, Shalom-Paz E, Shehata F, Al-Talib A, Tulandi T. Effects of excision of ovarian endometrioma on the antral follicle count and collected oocytes for in vitro fertilization. Fertil Steril 2010; 94:2340-2342.

Almog B, Shehata F, Sheizaf B, Tan SL, Tulandi T. Effects of ovarian endometrioma on the number of oocytes retrieved for in vitro fertilization. Fertil Steril 201 I;95:525-527.

Benaglia L, Somigliana E, Vercellini P, Abbiati A, Ragni G, Fedele L. Endometriotic ovarian cysts negatively affect the rate of spontaneous ovulation. Hum Reprod 2009;24:2183-2186.

Broekmans FJ, Kwee J, Hendriks DJ, Mol BW, Lambalk CB. A systematic review of tests predicting ovarian reserve and IVF outcome. Hum Reprod Update 2006; 12:685-7|8.
Busacca M, Riparini J, Somigliana E, Oggioni G, Izzo S, Vignali M, Candiani M. Postsurgical ovarian failure after laparoscopic excision of bilateral endomatriomas. Am J Obstet Gynecol 2006; 195:42I -425.

Candiani M, Barbieri M, Bottani B, Bertulessi C, Vignali M, Agnoli B, Somigliana E, Busacca M. Ovarian recovery after laparoscopic enucleation of ovarian cysts: insights from echographic short-term postsurgical follow-up. J Minim Invasive Gynecol 2005; 1 2:409-4I4.

Chang HJ, Han SH, Lee JR, Jee BC, Lee BI, Suh CS, Kim SH. Impact of laparoscopic cystectomy on ovarian reserve: serial changes of serum anti-Müllerian hormone levels. Fertil Steril 2010;94:343-349.

Demirol A, Guven S, Baykal C, Gurgan T. Effect of endometrioma cystectomy on IVF outcome: a prospective randomized study. Reprod Biomed Online 2006; I 2:639-643.

Di Prospero F, Micucci G. Is operative laparoscopy safe in ovarian endometriosis? Reprod Biomed Online 2009; 18:167.

Duru NK, Dede M, Acikel CH, Keskin U, Fidan U, Baser I. Outcome of in vitro fertilization and ovarian response after endometrioma stripping at laparoscopy and laparotomy. J Reprod Med 2007;52:805-809.

Esinler I, Bazdag G, Aybar F, Bayar U, Yarali H. Outcome of in vitro fertilization/intracytoplasmic sperm injection after laparoscopic cystectomy for endometriomas. Fertil Steril 2006;85:1730-1735.

Eskenazi B, Warner M, Bonsignore L, Olive D, Samuels S, Vercellini P. Validation study of nonsurgical diagnosis of endometriosis. Fertil Steril 2001;76:929-935.

Garcia-Velasco JA, Somigliana E. Management of endometriomas in women requiring IVF: to touch or not to touch. Hum Reprod 2009; 24:496-50I.

Garcia-Velasco JA, Mahutte NG, Corona J, Zuniga V, Giles J, Arici A, Pellicier A. Removal of endometriomas before in vitro fertilization does not improve fertility outcomes: a matched, case-control study. Fertil Steril 2004;8 I: I 194-1 197.

Gupta S, Agarwal A, Agarwal R, Loret de Mola JR. Impact of ovarian endometrioma on assisted reproduction outcomes. Reprod Biomed Online 2006; 1 3:349-360.

Ho HY, Lee RK, Hwu YM, Lin MH, Su JT, Tsai YC. Poor response of ovaries with endometrioma previously treated with cystectomy to controlled ovarian hyperstimulation. J Assist Reprod Genet 2002; |9:507-51।.

Horikawa T, Nakagawa K, Ohgi S, Kojima R, Nakashima A, Ito M, Takahashi $Y$, Saito $H$. The frequency of ovulation from the affected ovary decreases following laparoscopic cystectomy in infertile women with unilateral endometrioma during a natural cycle. J Assist Reprod Genet 2008;25:239-244.

Kennedy S, Bergqvist A, Chapron C, D'Hooghe T, Dunselman G, Greb R, Hummelshoj L, Prentice A, Saridogan E, ESHRE Special Interest Group for Endometriosis Endometrium Guideline Development Group. ESHRE Special Interest Group for Endometriosis and Endometrium Guideline Development Group. ESHRE guideline for the diagnosis and treatment of endometriosis. Hum Reprod 2005;20:2698-2704.

Kurjak A, Kupesic S. Scoring system for prediction of ovarian endometriosis based on transvaginal color and pulsed Doppler sonography. Fertil Steril 1994;62:8I-88.

Lan KC, Huang FJ, Lin YC, Kung FT, Lan TH, Chang SY. Significantly superior response in the right ovary compared with the left ovary after stimulation with follicle-stimulating hormone in a pituitary down-regulation regimen. Fertil Steril 2010;93:2269-2273.

Loh FH, Tan AT, Kumar J, Ng SC. Ovarian response after laparoscopic ovarian cystectomy for endometriotic cysts in 132 monitored cycles. Fertil Steril 1999;72:316-321.

Mais V, Guerriero S, Ajossa S, Angiolucci M, Paoletti AM, Melis GB. The efficiency of transvaginal ultrasonography in the diagnosis of endometrioma. Fertil Steril 1993;60:776-780. 
Maneschi F, Marasa L, Incandela S, Mazzarese M, Zupi E. Ovarian cortex surrounding benign neoplasms: a histologic study. Am J Obstet Gynecol 1993; 169:388-393.

Nelson SM, Yates RW, Lyall H, Jamieson M, Traynor I, Gaudoin M, Mitchell P, Ambrose P, Fleming R. Anti-Müllerian hormone-based approach to controlled ovarian stimulation for assisted conception. Hum Reprod 2009;24:867-875.

Ragni G, Somigliana E, Benedetti F, Paffoni A, Vegetti W, Restelli L, Crosignani PG. Damage to ovarian reserve associated with laparoscopic excision of endometriomas: a quantitative rather than a qualitative injury. Am J Obstet Gynecol 2005; 19:1908-1914.

Savelli L. Transvaginal sonography for the assessment of ovarian and pelvic endometriosis: how deep is our understanding? Ultrasound Obstet Gynecol 2009;33:497-501.
Somigliana E, Ragni G, Benedetti F, Borroni R, Vegetti W, Crosignani P. Does laparoscopic excision of endometriotic ovarian cysts significantly affect ovarian reserve? Insights from IVF cycles. Hum Reprod 2003; | 8:2450-2453.

Somigliana E, Infantino M, Benedetti F, Arnoldi M, Calanna G, Ragni G. The presence of ovarian endometriomas is associated with a reduced responsiveness to gonadotropins. Fertil Steril 2006; 86: $192-196$.

Somigliana E, Arnoldi M, Benaglia L, lemmello R, Nicolosi AE, Ragni G. IVF-ICSI outcome in women operated on for bilateral endometriomas. Hum Reprod 2008;23:1526-1530.

Vigano P, Somigliana E, Parazzini F, Vercellini P. Bias versus causality: interpreting recent evidence of association between endometriosis and ovarian cancer. Fertil Steril 2007;88:588-593. 